Reiter, Herwig/Witzel, Andreas (2021) Das fokussierte Interview. SocArXiv. doi:10.31235/osf.io/598wu.

\title{
Das fokussierte Interview
}

Reiter, Herwig ${ }^{1} \&$ Witzel, Andreas ${ }^{2}$

August 2021

\section{Zusammenfassung}

Der Artikel beschreibt Hintergrund und Methodik des fokussierten Interviews.

\section{Summary}

The article describes background and methodology of the Focused Interview.

\section{Vorgeschichte und Hintergrund ${ }^{3}$}

Die Entwicklung des fokussierten Interviews als Verfahren zur Befragung von Individuen, allein und in Gruppen, hat ihren komplexen und bislang wenig gewürdigten Ursprung im Forschungsansatz von Herta Herzog, der eigentlichen „Mutter der Fokusgruppe“ (Simonson 2016: 70), und in ihren empirischen Arbeiten zur Publikumsforschung - zuerst im Rahmen ihrer Promotion u.a. bei Paul Lazarsfeld in Wien Anfang der 1930er Jahre und später als seine Kollegin (und Ehefrau, 1937-1945) am Office of Radio Research in Princeton bzw. Columbia (Klaus 2008, Kleining 2016). Als Lazarsfeld im Jahr 1941 Robert Merton begegnete, involvierte er inn in seine und Herzogs Wirkungsforschung zu Kriegspropaganda mithilfe des sog. Lazarsfeld-Stanton Program Analyzers, einem Gerät zur automatisierten Erfassung spontaner Reaktionen bei der Betrachtung von Objekten und Filmen. Lazarsfeld übertrug Merton schließlich die Kodifizierung des dabei verwendeten Interviewverfahrens zur Ermittlung von Reaktionen auf unterschiedliche Stimuli und Testsituationen („post-exposure interviewing") (Levy 1982: 35; vgl. Hollonquist/Suchman 1944; Simonson 2005). Das fokussierte Interview wird erstmals in einem Kongressbeitrag und Artikel (Lazarsfeld/Merton 1968/1943: 570) diskutiert und danach von Merton gemeinsam mit Patricia Kendall (Lazarsfelds Ehefrau ab 1949) ausgearbeitet - mit unterschiedlichen Akzentsetzungen zunächst in einem Zeitschriftenartikel (Merton/Kendall 1946/1979) und dann als Monographie (Merton et al. 1990/1956). ${ }^{4}$ Seither werden das fokussierte Interview und die daraus hervorgegangenen Verfahren des Fokusgruppeninterviews und der Fokusgruppendiskussion in vielen Bereichen der Markt-, Medien- und Sozialforschung angewendet (Morgan 2001; Lee 2010; Morgan 2019). Der gemeinsame Nenner unterschiedlicher Verwendungen ist der (offen gelegte) Zweck der Anpassung der Stimulussituation aufgrund der Forschungsergebnisse (Merton et al. 1990/1956: 171-172).

\footnotetext{
${ }^{1}$ DJI - Deutsches Jugendinstitut e.V., Zentrum für Dauerbeobachtung und Methoden, München, Deutschland, reiter@dji.de

${ }^{2}$ Universität Bremen, im Ruhestand

${ }^{3}$ Dieser Text wurde als Handbuchartikel vorbereitet. Für eine umfassende Einführung siehe Merton et al. (1990/1956).

${ }^{4}$ In unserer Darstellung folgen wir bei Unterschieden der Monographie (Merton et al. 1990/1956) als der aktuelleren Quelle.
} 


\section{Beschreibung der Methodik}

Das fokussierte Interview ist ein Interviewverfahren zur Ermittlung von Reaktionen auf vorab analysierte Situationen. Im Unterschied zu Interviewformen, die Auskünfte zu vergleichsweise unspezifischen, aber thematisch umfänglichen und komplexen Erfahrungen (z.B. Lebensgeschichten, Alltagserfahrungen etc.) sammeln, geht es bei fokussierten Interviews um die Wirkung sehr eng definierter, natürlicher und künstlicher Testsituationen (auch: ,Stimulussituationen; z.B. Filme, Rundfunkprogramme, Artikel, Bücher, Experimente, soziale Situationen).

Die als „objektiv“ definierten „Merkmale“ (Merton et al. 1990/1956: 21) dieser Situationen und Stimuli werden vor dem Interview im Rahmen einer umfassenden Inhaltsanalyse erarbeitet und bilden die Grundlage für Hypothesen zur antizipierten Wirkungsweise bestimmter situationsbezogener Aspekte. Daraus ergeben sich Themen für einen Interviewleitfaden mit Hauptbereichen der Untersuchung und forschungsleitende Hypothesen zur gezielten Erhebung dafür relevanter Daten. Das eigentliche Interviewgespräch verknüpft diese thematische Engführung mit offenen Fragestrategien zur Sammlung subjektiver Definitionen der vorab analysierten Situation. Anhand möglichst vollständiger Berichte über die Erfahrungen der Situation besteht das Ziel in der Validierung von Hypothesen aus der Inhaltsanalyse der Situation bzw. der Bildung und weiterführenden Prüfung neuer Hypothesen. Konkret geht es um die Entdeckung von: bedeutsamen Aspekten der Testsituation; Widersprüchen zwischen antizipierten und tatsächlichen Wirkungen dieser Situationen; Reaktionen unterschiedlicher Subgruppen der Population darauf; und Prozessen in Verbindung mit experimentell erzeugten Wirkungen.

Fokussierte Interviews sind getragen von der Annahme (und primären Relevanzsetzung der Forschenden), dass die Teilnahme an der Testsituation grundsätzlich bedeutsame Reaktionen generiert, deren Details im Anschluss daran mithilfe darauf fokussierter Interviewkommunikation gesammelt werden können. Die Offenheit von fokussierten Interviews beschränkt sich auf fokussierte Mikrorelevanzsetzungen der Befragten im Sinne von Antworten auf offene Fragen zu vorgegebenen Themen. ${ }^{5}$ Ziel des Gesprächs ist die Förderung eines Erinnerungs- und Vergegenwärtigungsprozesses anhand „retrospektiver Introspektion“ (Merton et al. 1990/1956: 2139). Gemeint ist damit die möglichst ausführliche Schilderung der ins Gedächtnis zurückgerufenen Reaktionen zum Zeitpunkt der Erfahrung der Testsituation. Das Problem der Abgrenzung dieser retrospektiven Rekonstruktionen von Beschreibungen bzw. Interpretationen aus der gegenwärtigen Sicht wird dabei deutlich.

Mithilfe nicht-direktiver Fragetechniken nach dem Vorbild von Roethlisberger/Dickson (1939) und Rogers (1942) und der Verwendung von Erinnerungshilfen (z.B. Fotos, Ausschnitte der Situation) werden auch scheinbar nebensächliche Details der ursprünglichen Erfahrung der Testsituation auf eine Weise im Interview erfasst, die den analytisch unterschiedenen Kriterien des fokussierten Interviews von Umfang und Spektrum (range), Spezifiziät (specificity), Tiefe (depth), und persönlichem Hintergrund (personal context) gerecht werden soll. Ein gelungenes fokussiertes Interview sammelt möglichst viele und vielfältige Daten zu relevanten Aspekten; es produziert hoch

\footnotetext{
${ }^{5}$ Die explizite Einschränkung von Offenheit ist dem Festhalten an theoretischen Vorgaben geschuldet und macht sich in der Haltung gegenüber spontanen Relevanzsetzungen der Befragten geltend. So beschreiben Merton et al. (1990/1956: 134) dies angesichts der Gefahr des Abschweifens im Rahmen der Exploration des persönlichen Hintergrunds von Gesprächspartner*innen folgendermaßen: "The interviewer should remember that, in a focused interview, the interest is in those matters which are, or can be, related to the stimulus situation. Interest in discovering personal contexts should not be permitted to result in prolonged digressions; these should be brought back in focus by relating the interviewee's prior experiences, dispositions and status to his definition of the particular situation."
} 
spezifische und differenzierte Beschreibungen der erfahrenen Testsituation; es bringt damit verbundene affektive, kognitive und evaluative Bedeutungen hervor; und es erfasst die Hintergründe konkreter Bedeutungszuschreibungen. Empfehlungen zur Gesprächsführung in fokussierten Interviews beziehen sich auf alle vier Aspekte. Insbesondere die Vorschläge zur Vermeidung von Fehlern im Streben nach thematischer Breite (range) durch das starre Festhalten an Vorgaben aus dem Interviewleitfaden (Merton et al. 1990/1956: 43-53) veranlassten Hopf (1978) zur Ausarbeitung ihres Konzepts der „Leitfadenbürokratie“.

Die Vermischung von Aspekten von Einzel- und Gruppenerhebungsverfahren war von Anfang an Bestandteil des Ansatzes; allerdings standen Einzelansichten zunächst im Vordergrund (Hollonquist/Suchman 1944: 318-319). Fokussierte Interviews mit Gruppen sind vor allem dann vorteilhaft, wenn nach vielfältigen Situationsdefinitionen zahlreicher Personen gesucht wird. In ihren rudimentären Überlegungen zum Gruppeninterview (und unter Vernachlässigung des namengebenden Aspekts der Fokussierung) sehen Merton et al. (1990/1956: 135-169) neben pragmatischen Vorteilen weitere darin, dass die Gruppendynamik Zurückhaltung reduzieren, das Spektrum an Antworten erweitern und die Erinnerung von Details begünstigen könne.

\section{Gesamteinschätzung des Verfahrens}

Das für die 1940er Jahre innovative Verfahren des fokussierten Interviews ist ein frühes Dokument der Unzufriedenheit mit der Oberflächlichkeit herkömmlicher quantitativ-experimenteller Ansätze. Im historischen Rückblick stellt es einen ersten wichtigen, allerdings weniger wissenschaftstheoretisch fundierten als pragmatischen Schritt zur Emanzipation qualitativer Herangehensweisen dar.

Sein Anspruch besteht darin, im Kontext normativ-testender Forschungsansätze verbleibend, als „zusätzliches Hilfsmittel“ (Merton/Kendall 1979/1946: 177) zu fungieren, um die von den Autoren bemängelten, vielfach spekulativen Hypothesen und „Ad hoc Interpretationen“ mithilfe der Interpretation der Introspektion der Befragten im Interviewprozess zu validieren, d. h. zu ergänzen und zu korrigieren. Dieser Prozess bleibt theoretisch an die verwendeten Hypothesen und thematisch an die bezogen auf die "objektive" Situation inhaltsanalytisch abgeleitete Leitfadenstruktur gebunden. Die grundsätzliche Orientierung an Vorwissen macht das Verfahren im Prinzip anschlussfähig an die moderneren Mixed Methods Ansätze. Abweichende oder neue Aspekte außerhalb der theoretischen Vorgaben, sowohl im Interview selbst als auch in der anschließenden Auswertungsphase, drohen dabei allerdings aus dem Blick zu geraten. Das liegt daran, dass die entsprechend der Logik der Interviewtechnik resultierenden Frageformen (mit unterschiedlichem Strukturierungsgrad) in erster Linie das Ziel verfolgen, ausreichende Daten (z. B. für die Hypothesenprüfung) zu sichern und die Befragten auf die ,objektiven'Aspekte der Stimulussituation zu fokussieren und ggf. darauf zurückzuführen. Die gleichzeitig propagierte nondirektive Gesprächsführung und das variable und explizite Eingehen des Interviewenden auf die Ausführungen der Befragten haben das Ziel, möglichst auch nicht-antizipierte Reaktionen zu erfassen. Diese deutliche Erweiterung des Gestaltungsspielraums für die Befragten lässt eine Tendenz zu einer eher induktiven Orientierung des Erkenntnisprozesses im Interview erkennen, die - innovativ angesichts des damaligen wissenschaftstheoretischen Mainstreams - der heutigen Auffassung „Offenheitsprinzips“ in der qualitativen Sozialforschung nahekommt. 


\section{Literatur}

Hollonquist, Tore/Suchman, Edward A. (1944): Listening to the listener: experiences with the Lazarsfeld-Stanton Program Analyzer. In: Lazarsfeld, Paul F./Stanton, Frank N. (Hrsg.): Radio research 1942-1943. New York: Essential Books, War Edition. S. 265-334.

Klaus, Elisabeth (2008): What do we really know about Herta Herzog? - Eine Spurensuche. In: Medien \& Kommunikationswissenschaft 56, 2, 227-252.

Kleining, Gerhard (2016): The discovery process in Herta Herzog's research on radio daytime serials. With an appendix on the invention of the focus groups. In: Klaus, Elisabeth/Seethaler, Josef (Hrsg.): What do we really know about Herta Herzog? Frankfurt: PL Academic Research. S. 117135.

Lazarsfeld Paul F./Merton Robert K. (1968/1943): Studies in radio propaganda. In: Transactions of the New York Academy of Sciences, Vol.6, Issue 2, Series II, 58-74. (Zitiert in: Merton, Robert K. (1968): Social theory and social structure. New York: The Free Press. 563-582.)

Lee, Raymond M. (2010): The secret life of focus groups: Robert Merton and the diffusion of a research method. In: The American Sociologist, 41, 2, 115-141.

Levy, Mark, R. (1982): The Lazarsfeld-Stanton program analyzer: a historical note. In: Journal of Communication, 32, 4, 30-38.

Merton, Robert K./Kendall, Patricia L. (1979/1946): Das fokussierte Interview. In: Hopf, Christel/Weingarten, Elmar (Hrsg.): Qualitative Sozialforschung. Stuttgart: Klett-Kotta. S. 171-204.

Merton, Robert K./Fiske, Majorie/Kendall, Patricia L. (1990/1956): The focused interview. A manual of problems and procedures. Second edition. New York: The Free Press.

Morgan, David L. (2001): Focus group interviewing. In: Gubrium, Jaber F./Holstein James A. (Hrsg.): Handbook of interview research. Context \& method. Thousand Oaks: Sage. S. 141-159.

Morgan, David, L. (2019): Basic and advanced focus groups. Thousand Oaks: Sage.

Roethlisberger, Fritz J./Dickson, William J. (1939): Management and the worker. Cambridge: Harvard University Press.

Rogers, Carl R. (1942): Counseling and psychotherapy: new concepts in practice. Boston: Houghton Mifflin Company.

Simonson, Peter (2005): The serendipidity of Merton's communications research. In: International Journal of Public Opinion Research. 17, 3, 277-297.

Simonson, Peter (2016): Herta Herzog and the founding mothers of mass communication research. In: Klaus, Elisabeth/Seethaler, Josef (Hrsg.): What do we really know about Herta Herzog? Frankfurt: PL Academic Research. S. 61-83. 